\title{
Vigilância epidemiológica no Programa de Controle da Doença de Chagas em Minas Gerais, Brasil (1984-1998)
}

\author{
Epidemiological surveillance in the Chagas \\ disease control program in Minas Gerais \\ State, Brazil (1984-1998)
}

Elizabeth Castro Moreno 1

Lúcia Baracho 1

\footnotetext{
1 Fundação Nacional de Saúde, Coordenação Regional de Minas Gerais. Rua Espírito Santo 500, Belo Horizonte, $M G$ 30160-030, Brasil.
}

\begin{abstract}
This paper describes the surveillance phase of the Chagas Disease Control Program in Minas Gerais State. Surveillance was conducted by the County Health Services with community participation in the planning, decision-making, and maintenance processes and is intended to be sustainable in the context of Brazil's Unified National Health System (SUS).

Key words Chagas Disease; Triatominae; Epidemiological Surveillance; Vector Control; Prevention and Control
\end{abstract}

Resumo É apresentada a metodologia utilizada em Minas Gerais para a vigilância no Programa de Controle da Doença de Chagas. O sistema é desenvolvido com a participação das comunidades, desde o planejamento à tomada de decisões e deve ser gradativamente assumido pelos municípios no processo de municipalização das ações e serviços de saúde do Sistema Único de Saúde (SUS).

Palavras-chave Doença de Chagas; Triatominae; Vigilância Epidemiológica; Controle de Vetores; Prevenção e Controle 


\section{Introdução}

A vigilância no Programa de Controle da Doença de Chagas (PCDCh) foi idealizada como uma etapa de um programa tradicionalmente vertical, realizado pela Fundação Nacional de Saúde (FNS), baseado em ações de pesquisa (busca ativa de triatomíneos) e em borrifação de domicílios. Tradicionalmente, a fase de vigilância entomológica era instalada em municípios que apresentassem índices de dispersão de triatomíneos abaixo de 5\%. Eram desenvolvidos dois tipos de atividades: vigilância ativa, pesquisa de focos de triatomíneos, realizada de casa em casa por turmas de agentes de saúde pública; e vigilância passiva, visita mensal aos Postos de Informação de Triatomíneos (PITs) para recolhimento de notificações e trabalho de divulgação dos PITs, feito de casa em casa (MS, 1980).

Na década de 80, com a expansão do PCDCh em todo o país, iniciaram-se as discussões com o objetivo de desenvolver uma metodologia mais adequada para a condução da vigilância. Foi elaborada, em Minas Gerais, uma nova proposta baseada na experiência de participação comunitária no Município de Bambuí (Dias \& Garcia, 1978). Sua implantação deu-se gradativamente, a partir de 1986, no estado, acompanhando o processo de implantação das Ações Integradas de Saúde e, posteriormente, do Sistema Único de Saúde.

\section{Metodologia}

A definição sobre onde, quando e quem implantar a vigilância depende da realidade de cada região, mas alguns procedimentos básicos podem nortear o planejamento do sistema mais adequado a cada situação.

A indicação de municípios para a implantação da vigilância é feita após a avaliação das atividades de controle já desenvolvidas no município, através da análise da série histórica do programa (MS, 1980). Os principais indicadores utilizados nessa avaliação são: ausência de Triatoma infestans, decréscimo progressivo e significativo dos índices entomológicos, decréscimo da densidade triatomínica, redução do número de ninfas no intradomicílio, situação epidemiológica dos municípios limítrofes e projeção de notificações anuais. As informações são mapeadas e resumidas, com o objetivo de torná-las acessíveis aos municípios, já que a vigilância deixa de ser uma atividade exercida exclusivamente pela FNS.

Foram definidas duas formas de condução do trabalho: vigilância "epidemiológica" e a “institucional”. A vigilância epidemiológica consiste na definição de um agente de saúde da FNS ou do município. Essa pessoa é responsável pela orientação e mobilização da comunidade e pelo atendimento imediato das notificações de triatomíneos, através de pesquisa e borrifação das casas notificadas. O encaminhamento das notificações continua sendo feito através de PITs, instalados nas localidades rurais e sede dos municípios. Entretanto, procura-se valorizar ao máximo a participação dos representantes formais e líderes naturais da comunidade nas decisões e no planejamento das atividades.

Para a implantação da vigilância epidemiológica, são realizadas reuniões com representantes formais do município (Conselho Municipal de Saúde, profissionais de saúde e ensino) para avaliação dos resultados já alcançados e apresentação dos objetivos e das propostas do trabalho. São realizadas reuniões em todas as localidades de maior referência para a população e onde sempre persistiu a ocorrência de triatomíneos, a fim de obter a indicação dos locais mais adequados para a instalação dos PITs. É dada preferência aos postos de saúde e às escolas, ou, na ausência destes, residências de moradores indicados pela comunidade. As notificações são encaminhadas a um PIT central, na sede do município. O levantamento da infra-estrutura básica das áreas de saúde e ensino permite a definição das estratégias mais adequadas para a estruturação da vigilância no município.

O agente de vigilância é responsável pelo recolhimento e atendimento das notificações em um grupo de municípios vizinhos, através da visita ao PIT central e, eventualmente, aos PITs rurais. Sempre que necessário, são programadas atividades educativas com a comunidade, como demonstrações de triatomíneos e de sua captura, mutirões para limpeza de quintais, palestras, seminários, oficinas, exposições e reuniões, com o objetivo de conscientizar a população sobre a situação local e orientar o encaminhamento das notificações. É realizada pesquisa para confirmação da existência de foco em todas as casas notificadas, sendo feita a borrifação do domicílio, independente do encontro de triatomíneos pelo agente de saúde.

A devolução de dados para a comunidade é realizada através de reuniões periódicas e contatos informais com os colaboradores e instituições locais. A participação da comunidade é avaliada através do comparecimento às reuniões, decisões tomadas, sugestões e produção de material informativo e solicitações do agente de saúde para realização de atividades edu- 
cativas. A produtividade dos PITs é avaliada de acordo com a expectativa de incidência de triatomíneos na área e capacidade da população em diferenciar os triatomíneos de outros insetos.

Em municípios onde as ações de saúde estão sendo municipalizadas, as atividades de controle de outras endemias tradicionalmente realizadas pela FNS estão sendo desenvolvidas por equipes municipais, treinadas e coordenadas pelo agente de saúde responsável pela vigilância do PCDCh. Na medida do possível, são desenvolvidas também outras ações de saúde desenvolvidas pelas diretorias regionais de saúde e pelos municípios.

A vigilância institucional consiste na definição de uma instituição de saúde do município como referência para a comunidade a fim de que atue no recebimento de notificações e no encaminhamento ao agente de vigilância do município mais próximo para o atendimento da notificação. Se necessário, a instituição programa atividades educativas voltadas para o problema local. Essa instituição de referência, os postos de saúde e escolas são visitados a cada três meses, aproximadamente, em função da grande rotatividade dos profissionais municipais.

A vigilância institucional é implantada em municípios que atendam a alguns dos seguintes critérios: 1) negativos desde o início do programa; 2) predominância de avaliações (Avs) negativas; 3) índices de infestação domiciliar menor que $1 \%$ nas três últimas Avs; 4) infra-estrutura e organização dos serviços de saúde; 5) extensão territorial menor que $200 \mathrm{~m}^{2}$.

Em todas as duas formas de trabalho são realizados treinamentos formais, por município, para os responsáveis pelos PITs e equipes institucionais. O sucesso da vigilância depende, em grande parte, da seleção e da capacitação dos agentes de saúde, que devem estar aptos a desenvolver tais treinamentos e as atividades de envolvimento comunitário.

A manutenção de um sistema de informações garante o acompanhamento periódico do trabalho, necessário para avaliação dos resultados. Foi estruturado um banco de dados, que permite aos níveis interessados (municipal, estadual ou federal) o acesso às informações. A detecção precoce de alterações nas tendências observadas, como aumento dos índices entomológicos e substituição de espécies, permite rever a necessidade de mudanças nas estratégias de controle e vigilância.

Paralelamente ao trabalho de vigilância, têm sido realizados inquéritos sorológicos em escolares de 7 a 14 anos residentes na zona rural desses municípios, com o objetivo de avaliar a eficácia do PCDCh na redução da infecção humana pelo Trypanosoma cruzi (MS, 1984a). São realizadas colheitas de sangue em papel filtro e, quando necessário, colheita de sangue venoso para confirmação dos resultados. As crianças infectadas são encaminhadas a médicos de referência, para avaliação, acompanhamento e tratamento.

Atualmente, a vigilância não é mais considerada uma fase e está sendo implantada em todos os municípios do Estado, conforme as novas diretrizes técnicas do programa (MS, 1984b). Com o processo de municipalização em curso, a vigilância de Chagas tende a ser gradativamente incorporada às atividades de vigilância em saúde das secretarias municipais. Caberá aos níveis regionais e centrais da FNS normatizar os procedimentos, capacitar as equipes institucionais, realizar acompanhamento/supervisão técnica e avaliar os resultados.

\section{Resultados}

Atualmente, 280 municípios estão sob vigilância em Minas Gerais, o que corresponde a $45,2 \%$ do total de municípios trabalhados pelo PCDCh no Estado. A avaliação parcial dos dados dos últimos cinco anos indica as seguintes tendências: - Os municípios com registro de notificações correspondem a $40 \%$ a $60 \%$ do total de municípios em vigilância no Estado. São recebidas em média mil notificações de triatomíneos anualmente. A maioria (80\%) das notificações é feita com um exemplar de triatomíneo adulto. Cerca de 55\% dos triatomíneos têm sido capturados no intradomicílio, e entre $80 \%$ a $90 \%$ das notificações não são confirmadas pela pesquisa posterior do agente de saúde pública. As espécies triatomínicas prevalentes e os respectivos índices de infecção pelo T. cruzi são: Panstrongylus megistus $(1,1 \%)$, Triatoma sordida (1,8\%), Rhodnius neglectus $(0,0 \%)$, Triatoma vitticeps (6,8\%), Panstrongylus diasi $(0,0 \%)$, Panstrongylus geniculatus (4,2\%) e Triatoma pseudomaculata (2,8\%).

- No período de 1989 a 1998, o inquérito sorológico foi realizado em 164 municípios em vigilância. Foram examinadas 38.101 amostras de sangue, sendo encontradas $15(0,04 \%)$ crianças entre 7 e 14 anos, com infecção por T. cruzi.

\section{Conclusão}

Os indicativos da interrupção da transmissão vetorial, como a diminuição dos índices entomológicos e da incidência de casos agudos da 
doença, apontam para a necessidade de se reforçar os sistemas de vigilância existentes, com o objetivo de assegurar os resultados já alcançados. Em Minas Gerais, os resultados dos últimos anos, sugerem que triatomíneos adultos invadem as casas, mas que apenas ocasionalmente formam colônias. A vigilância deve ter, portanto, um caráter permanente, a fim de assegurar a detecção precoce dos triatomíneos e sua eliminação.

A experiência desenvolvida em Minas Gerais deve ser adaptada e aprimorada de acordo com a realidade de cada região e incorporada aos sistemas municipais de vigilância em saúde.

\section{Referências}

DIAS, J. C. P. \& GARCIA, A. L. R., 1978. Vigilancia Epidemiológica con participación comunitaria. Un programa de enfermedad de Chagas. Boletín de la Oficina Sanitaria Panamericana, 84:533-544.

MS (Ministério da Saúde), 1980. Manual de Normas Técnicas da Campanha de Controle da Doença de Chagas. Brasília: Centro de Documentação do Ministério da Saúde.

MS (Ministério da Saúde), 1994a. Inquérito Sorológico para Avaliação do Programa de Controle da Doença de Chagas. Normas Técnicas. Brasília: MS.

MS (Ministério da Saúde), 1994b. Controle da Doença de Chagas - Diretrizes Técnicas. Brasília: MS. 